This is the accepted manuscript of:

Raschi E, Poluzzi E, Marchesini G, De Ponti F.

Dapagliflozin and cardiovascular outcomes: anything else to DECLARE?

Expert Opin Pharmacother. 2019 Jun;20(9):1087-1090

Final peer reviewed version available: $\underline{10.1080 / 14656566.2019 .1598970}$

Rights / License:

The terms and conditions for the reuse of this version of the manuscript are specified in the publishing policy. For all terms of use and more information see the publisher's website.

This item was downloaded from IRIS Università di Bologna (https://cris.unibo.it/)

When citing, please refer to the published version. 


\title{
Dapagliflozin and Cardiovascular Outcomes: anything else to DECLARE?
}

\begin{abstract}
Introduction: People with type 2 diabetes mellitus (T2DM) are at increased cardiovascular risk and regulatory approach impose current antidiabetes drugs to be safety from a cardiovascular standpoint.

Areas covered: In this paper, the authors critically discussed the background, trial design, results and implications of a recent cardiovascular outcome trial (CVOT) [NCT01730534; DECLARE-TIMI 58 study], which demonstrated that dapagliflozin was non-inferiority to placebo in terms of major adverse cardiovascular events, and superior for the occurrence of hospitalization for heart failure (HF) and composite real endpoint, thus confirming the cardiovascular benefit of sodium-glucose co-transporter-2 (SGLT2) inhibitors. No statistically-significant imbalances were found for amputations, fractures, and stroke (debated safety issues emerged in previous CVOTs).

Expert opinion: DECLARE-TIMI 58 is the longest (4.2 years of follow up), largest ( $>17,000$ patients) and broadest ( $40 \%$ of patients with established atherosclerotic cardiovascular disease) CVOT raising the debate towards tailored therapy in primary prevention and the potential use of SGLT2 inhibitors in patients with HF without T2DM.
\end{abstract}

Keywords: dapagliflozin, sodium-glucose co-transporter-2 inhibitor, cardiovascular outcome trial, major adverse cardiovascular events, heart failure, type 2 diabetes

Word count: 1520

Number of references: 11

Number of figures: 1

Supplementary Material: 1 


\section{DECLARE-TIMI 58 as a paradigm of a pragmatic adaptive trial design}

Results from the Dapagliflozin Effect on Cardiovascular Events-Thrombolysis in Myocardial Infarction 58 (DECLARE-TIMI 58) trial have been recently published in the New England Journal of Medicine, fulfilling great expectations: the effects of dapagliflozin, a sodium-glucose co-transporter-2 (SGLT2) inhibitor, were tested on cardiovascular and renal outcomes in a broad population of people with type 2 diabetes mellitus (T2DM) and established atherosclerotic cardiovascular disease (ASCVD) or multiple risk factors for ASCVD [1].

This randomized, double-blind, multinational, placebo-controlled, phase 3 trial was originally designed to demonstrate the non-inferiority of dapagliflozin with a primary safety outcome of major adverse cardiovascular events (MACE), as recommended by regulatory guidelines. However, in accordance with the modern principle of adaptive trial design, compelling data from the EMPA-REG OUTCOME trial [2] with respect to cardiovascular death and hospitalization for heart failure (HHF), as compared to MACE, prompted the executive committee to amend the protocol (before viewing data on MACE from DECLARETIMI 58) to include two primary efficacy, superiority outcomes: MACE and the combination of cardiovascular death or HHF. This change was notified to regulators, and patients signed a revised informed consent also describing positive results of the EMPA-REG OUTCOME trial. Two secondary efficacy outcomes were prespecified: death from any cause and a renal composite outcome, defined as a sustained decrease of $40 \%$ or more in estimated glomerular filtration rate (eGFR) - calculated by means of the Chronic Kidney Disease Epidemiology Collaboration equation - to less than $60 \mathrm{ml}$ per minute per $1.73 \mathrm{~m}^{2}$ of body-surface area, incident end-stage renal disease, or death from renal or cardiovascular causes.

Eligible patients were $\geq 40$ years with T2DM, had a glycated hemoglobin level of $6.5-12.0 \%$, and a creatinine clearance $\geq 60 \mathrm{ml} / \mathrm{min}$. They were first enrolled in a 4-to-8-week, single-blind run-in placebo period, and eventually randomly assigned, in a double-blind fashion, to receive dapagliflozin (10 $\mathrm{mg} /$ day) or matching placebo. The use of other glucose-lowering agents (other than an open-label SGLT2 inhibitor, pioglitazone, or rosiglitazone) was at the discretion of the treating physician, simulating a real-world pragmatic scenario. Patients were followed-up every 6 months until trial completion, including phone contact every 3 months between in-person visits. Adjudicated events were analyzed according to the intention-to-treat principle.

\section{Key findings to DECLARE}

A total of 17,160 participants completed the run-in phase (of 25,698 initially-enrolled participants), including 6,974 patients (40.6\%) with established ASCVD and 10,186 (59.4\%) with multiple risk factors for ASCVD. During a median follow-up of 4.2 years, 3,962 patients dropped out: 1,811 (21.1\%) in the 
dapagliflozin group and 2,151 (25.1\%) in the placebo group. Rates of withdrawal of consent (224 patients, i.e., $0.3 \%$ per year) and loss to follow-up (30 patients, $<0.1 \%$ per year) were low and did not differ between groups. Baseline characteristics of patients were well balanced between the groups, with $10 \%$ of patients having a history of heart failure.

Dapagliflozin met the pre-specified criterion for non-inferiority with respect to MACE (upper boundary of the $95 \% \mathrm{Cl}<1.3 ; \mathrm{P}<0.001$ for noninferiority), as well as the efficacy composite endpoint of cardiovascular death or HHF (4.9\% vs. $5.8 \%$; hazard ratio:0.83; $95 \% \mathrm{Cl}: 0.73-0.95 ; \mathrm{P}=0.005)$. It should be noted that the lower rate of this composite outcome was however driven by a lower rate of HHF in the dapagliflozin group (hazard ratio:0.73; 95\% Cl:0.61-0.88), with no difference in the rate of cardiovascular death (hazard ratio:0.98; 95\% $\mathrm{Cl}: 0.82-1.17$ ). These data were similar in patients grouped according to the presence of established ASCVD vs. multiple risk factors for ACSVD. In the overall population, the incidence of the renal composite outcome was $4.3 \%$ in dapagliflozin vs $5.6 \%$ in placebo (hazard ratio:0.76; $95 \% \mathrm{Cl}: 0.67-0.87)$, whereas the rate of death from any cause $(6.2 \%$ vs $6.6 \%$; hazard ratio $=0.93 ; 95 \% \mathrm{Cl}=0.82$ 1.04), amputation, fractures and volume depletion were not different between groups. Diabetic ketoacidosis was more common in dapagliflozin users ( $0.3 \%$ vs $0.1 \%$; hazard ratio:2.18; $95 \% \mathrm{Cl}: 1.10-4.30$ ), as were genital infections leading to discontinuation (0.9\% vs. $0.1 \%$; hazard ratio:8.36; $95 \% \mathrm{Cl}: 4.19-16.68$ ), both in men and in women, although serious genital infections were rare (two events in each group). Six cases of Fournier's gangrene were reported (one with dapagliflozin group).

\section{Expert opinion}

DECLARE-TIMI 58 study is the longest (4.2 years), largest (>17,000 patients) and broadest cardiovascular outcome trial (CVOT) published so far on antidiabetic drugs [3]. The history of CV effects of SGLT2 inhibitors is paradigmatic. The EMPA-REG OUTCOME trial exclusively enrolled people with T2DM and established ASCVD (essentially secondary prevention), with substantial benefit of empagliflozin in terms of MACE, HHF and renal outcomes [2, 4]; later, the CANVAS program, reported data on a spectrum of individuals with T2DM (65\% with established ASCVD, a mix of primary and secondary prevention), and confirmed efficacy of canagliflozin in reducing the incidence of MACE, HHF, renal outcomes, not cardiovascular mortality (Figure 1) [5]. This may be related to the difference in time-to-effect between these trials: in the EMPA-REG OUTCOME trial the rate of the MACE, mortality and HHF between the empagliflozin and placebo groups rapidly diverged within the first 6 months after randomization, whereas in CANVAS program the divergence of the MACE (canagliflozin vs. placebo) was more gradual.

In the DECLARE-TIMI 58 trial only $40 \%$ of patients had established ASCVD; this may explain the lack of significant effects in the rates of MACE and cardiovascular death, the reduced extent of risk reduction in terms of HHF, and the divergence of incidence in cardiovascular death or HHF, which became evident only 
at month 18 (with slight increase), and after month 24 for composite renal outcomes. Therefore, DECLARETIMI 58 extends our knowledge of the cardiorenal effect of dapagliflozin in a real-world scenario of patients with cardiovascular risk factors or established ASCVD [6], in line with evidence recently provided by the observational pharmaco-epidemiological research (such as CVD-REAL studies), thus supporting a clinicallysignificant cardiovascular benefit of SGLT2 inhibitors in routine practice, especially in terms of HHF and renal endpoints [7]. Heart failure is probably the most common cardiac condition in T2DM; although it was not part of the primary composite of the MACE endpoint of these trials, the occurrence of HHF was remarkably reduced with SGLT2 inhibitors, irrespective of a previous history of heart failure at randomization, as supported by a systematic review with meta-analysis of CVOTs [8]. Of note, the number needed to treat to benefit for HHF was 116 for dapagliflozin in DECLARE-TIMI 58, as compared to 72 for empagliflozin in EMPAREG-OUTCOME. The glucosuric effect of SGLT2 inhibitors is unlikely to fully explain the observed benefit on HHF; several additional cardio-reno-vascular mechanisms have been proposed, including direct and indirect effects such as improvement of myocardial energy metabolism, inhibition of the sodium hydrogen exchange, increase in glucagon, inhibition of renal urate reuptake and relevant lower plasma uric acid, increase adenosine release causing renal vasodilation $[3,6]$.

Taken together, these findings open new avenues on the latest American Diabetes Association (ADA)-European Association for the Study of Diabetes (EASD) joint position statement [9] and American College of Cardiology (ACC) Expert Consensus Decision Pathway on Novel Therapies in T2DM for Cardiovascular Risk Reduction [10], which has prioritised the use of SGLT2 inhibitors only in people with ASCVD, heart failure, or non end-stage chronic kidney disease. The challenging questions is: is it time to extend their use to the broader population of patients with T2DM and multiple risk factors? To address this core clinical question, safety concerns should be also critically appraised (Figure 1, Supplementary Table for details). Notably, canagliflozin was associated with an increased incidence of fractures and atraumatic below-knee lower extremity amputation (BKA), as yet unexplained, and the DECLARE-TIMI 58 was awaited to verify whether these adverse effects should be considered as a class- rather than drug-specific toxicities. Overall, the trial confirmed that genital infections and ketoacidosis are strongly associated with SGLT2 inhibitors (expected from their mechanism of action), confirming they are common to all SGLT2 inhibitors, but no signal of BKA and fractures emerged, suggesting an off-target effect specific to canagliflozin.

In the light of current guidelines and position statement from ADA/EASD/ACC, the research agenda is now focused on: 1) the assessment of SGLT2 inhibitors on cardio-renal outcomes also in populations without T2DM, to verify the cardiovascular benefits in low-risk groups; 2) monitoring post-marketing rare unexpected safety concerns such as necrotizing fasciitis of the perineum, also known as Fournier's gangrene, as warned by the Food and Drug Administration; 3) comparing head-to-head SGLT2 inhibitors with other antidiabetic agents to clarify the optimal antidiabetic regimen, including dipeptidyl peptidase-4 (DPP4) inhibitors, with contrasting data on HHF, and glucagon-like peptide 1 receptor agonists (GLP1-RAs), 
the only antidiabetic class with positive data from relevant CVOTs [3]. While CVD-REAL studies have mainly used DPP4 inhibitors as comparators, a recent cohort study (nationwide registers from Sweden and Denmark) compared SGLT2 inhibitors with GLP1-RAs (the only antidiabetic class with positive data from relevant CVOTs), and confirmed the increased risk of lower limb amputations and diabetic ketoacidosis [11].

Next-generation CVOTs and other sources of real-world data (observational pharmacoepidemiological research and pharmacovigilance databases) are at the front line to (a) selecting SGLT2 inhibitors as first-line therapy after metformin in patients with T2DM, irrespective of the underlying cardiovascular phenotype, (b) unraveling the issue of a class effect.

\section{Funding}

This manuscript was not funded. Authors are supported by Institutional funds of the University of Bologna.

\section{Declaration of interest}

E Raschi, E Poluzzi, F De Ponti have no conflicts of interest relevant to the content of the present work. G Marchesini was involved in advisory boards from Gilead, Lilly, Astra-Zeneca, and took part in clinical studies on NAFLD and T2DM sponsored by Sanofi, Lilly, Novo, Janssen, Glaxo, Genfit, Gilead.

Figure 1. Overview of major findings of cardiovascular outcome trials (CVOTs) on SGLT2 inhibitors. In parenthesis percentage of patients with atherosclerotic cardiovascular disease (ASCVD).

MACE: major adverse cardiovascular events (please note that this definition may vary between studies); HHF: hospitalization for heart failure; BKA: atraumatic below-knee lower extremity amputation.

Please note that, in the EMPAREG-OUTCOME, a non-significant imbalance in the rates of stroke emerged. See Supplementary material for details on the various outcomes. 


\section{References}

Papers of special note have been highlighted as either of interest $(\bullet)$ or of considerable interest $(\bullet \bullet)$ to readers.

1. Wiviott et al. Dapagliflozin and Cardiovascular Outcomes in Type 2 Diabetes. N Engl J Med. 2018 Nov 10 doi: 10.1056/NEJMoa1812389 [Epub ahead of print]

-• The latest cardiovascular outcome trial on the SGLT2 inhibitor dapagliflozin

2. Zinman et al. Empagliflozin, cardiovascular outcomes, and mortality in type 2 diabetes. N Engl J Med 2015;373:2117-2128.

-• The first cardiovascular outcome trial on the SGLT2 inhibitor empafliflozin

3. Bailey CJ, Marx N. Cardiovascular protection in type 2 diabetes: Insights from recent outcome trials. Diabetes Obes Metab. 2018 Aug 8. doi: 10.1111/dom.13492. [Epub ahead of print]

4. Wannet et al. Empagliflozin and Progression of Kidney Disease in Type 2 Diabetes. N Engl J Med. 2016;375:323-334.

5. Neal et al. Canagliflozin and Cardiovascular and Renal Events in Type 2 Diabetes. N Engl J Med. 2017;377:644-657.

6. Zelniker TA, Braunwald E. Cardiac and Renal Effects of Sodium-Glucose Co-Transporter 2 Inhibitors in Diabetes. J Am Coll Cardiol. 2018;72:1845-1855.

7. Raschi et al. Observational research on sodium glucose co-transporter-2 inhibitors: A real breakthrough? Diabetes Obes Metab. 2018;20:2711-2723.

8. Zelniker et al. SGLT2 inhibitors for primary and secondary prevention of cardiovascular and renal outcomes in type 2 diabetes: a systematic review and meta-analysis of cardiovascular outcome trials. Lancet. 2018 Nov 9. pii: S0140-6736(18)32590-X. doi: 10.1016/S0140-6736(18)32590-X. [Epub ahead of print]

9. Davis et al. Management of Hyperglycemia in Type 2 Diabetes, 2018. A Consensus Report by the American Diabetes Association (ADA) and the European Association for the Study of Diabetes (EASD). Diabetes Care. 2018 Oct 4. pii: dci180033. doi: 10.2337/dci18-0033. [Epub ahead of print]

-• The latest Guideline on pharmacotherapy of people with diabetes

10. Das et al. 2018 ACC Expert Consensus Decision Pathway on Novel Therapies for Cardiovascular Risk Reduction in Patients With Type 2 Diabetes and Atherosclerotic Cardiovascular Disease: A Report of the American College of Cardiology Task Force on Expert Consensus Decision Pathways. J Am Coll Cardiol. 2018 Nov 26. pii: S0735-1097(18)38498-5. doi: 10.1016/j.jacc.2018.09.020. [Epub ahead of print]

-. The latest expert consensus decision pathway on pharmacotherapy of people with type 2 diabetes for cardiovascular risk reduction

11. Ueda et al. Sodium glucose cotransporter 2 inhibitors and risk of serious adverse events: nationwide register-based cohort study. BMJ. 2018 Nov 14;363:k4365. doi: 10.1136/bmj.k4365 


\section{EFFICACY OUTCOMES}

DEBATED SAFETY OUTCOMES

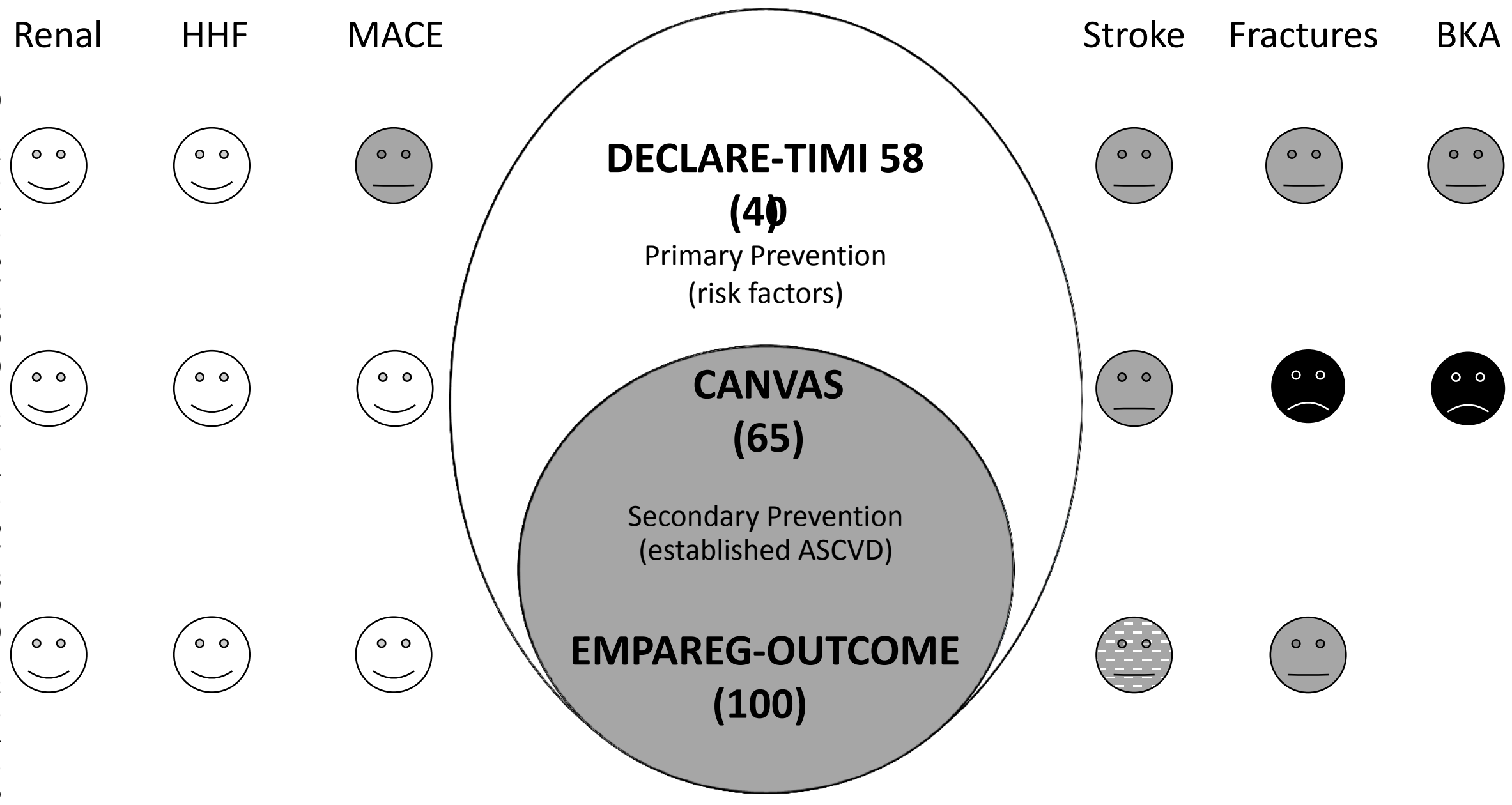


Overview of key features and major findings of cardiovascular outcome trials (CVOTs) on SGLT2 inhibitors. Numbers represent relative risks (reduced or increased) extracted from published CVOTs.

Colour coding: GREEN: significant reduced effect (benefit); RED: significant increased effect (risk); GREY: non-significant effect (benefit or risk).

$\uparrow=$ increased rate vs placebo; $\downarrow=$ reduced rate vs placebo; $\leftrightarrow=$ no difference vs placebo.

\begin{tabular}{|c|c|c|c|c|c|c|c|c|c|c|}
\hline & \multicolumn{4}{|c|}{ EFFICACY } & \multicolumn{6}{|c|}{ SAFETY } \\
\hline CVOT (\% with ASCVD) & MACE & CVM & HHF & Renal & GTI & DKA & Stroke & BKA & Fractures & Neoplasms \\
\hline EMPA-REG-OUTCOME (100) & $\downarrow 14$ & $\downarrow 38$ & $\downarrow 35$ & $\downarrow 39$ & 个* & $\leftrightarrow{ }^{*}$ & $\uparrow 24$ & & $\leftrightarrow{ }^{*}$ & \\
\hline CANVAS (65) & $\downarrow 14$ & $\downarrow 13$ & $\downarrow 37$ & $\downarrow 40$ & 个\# & $\leftrightarrow \#$ & $\downarrow 13$ & 个\# & 个\# & 个\# \\
\hline DECLARE-TMI 58 (40) & $\downarrow 7$ & $\downarrow 2$ & $\downarrow 17$ & $\downarrow 24$ & 个836 & 个218 & $\uparrow 1$ & $\uparrow 9$ & $\uparrow 4$ & $\downarrow 43$ \\
\hline
\end{tabular}

ASCVD: atherosclerotic cardiovascular disease; MACE: major adverse cardiovascular events (please note that this definition may vary between studies); CVM: cardiovascular mortality; HHF: hospitalization for heart failure; GTI: genital tract infections; DKA: diabetic ketoacidosis; BKA: atraumatic below-knee lower extremity amputation.

* For GTI: $6.4 \%$ vs $1.4 \%$ (P<0.001); for DKA: $<0.1 \%$ vs $0.1 \%$; for fractures: $3.8 \%$ vs $3.9 \%)$.

\# For GTI: $34.9 \%$ vs 10.8 in male and $68.8 \%$ vs $17.5 \%$ in women, $p<0.001$; for DKA: $0.6 \%$ vs $0.3 \%, p=0.14$; for BKA: $6.3 \%$ vs $3.4 \%$, $p<0.001$; for fractures: $15.4 \%$ vs $11,9 \%, p=0.02$; for neoplams: $0.6 \%$ vs $0.2 \%, p=0.17$ [renal], $1.0 \%$ vs $1.1 \%, p=0.74$ [bladder]; $3.1 \%$ vs $2.6 \%, p=0.65$ [breast]. 